\title{
Reconstrução da parede torácica nos defeitos adquiridos
}

\section{Chest wall reconstruction in acquired defects}

\author{
Marcus Vinicius H. de Carvalho"; Eduardo Baldassari Rebeis, TCBC-SP2; Evaldo Marchi ${ }^{3}$
}

\section{INTRODUÇÃO}

O tórax pode ser acometido por agentes agressores que, por si só, promovem deformidade da parede torácica. Isto ocorre, por exemplo, em decorrência de trauma com desalinhamento das estruturas ósseas e/ou perda de substância da parede torácica. Outro fator que pode deformar a parede do tórax é a infecção, que pode ocorrer nos tecidos lesionados pelo trauma ou se originar no tecido pulmonar ou no espaço pleural. A infecção pode resultar em defeito da parede torácica diretamente, ou processos cirúrgicos para tratamento da infecção podem deformar a parede (por exemplo, pleurostomia). Os defeitos da parede torácica também podem ser secundários a tumores, a procedimentos cirúrgicos com grandes ressecções ósseas e/ou musculares ou à irradiação para tratamento de neoplasias.

A reconstrução da parede torácica objetiva, fundamentalmente, restaurar a integridade da parede, conseguir dinâmica respiratória adequada e proteger órgãos vitais ${ }^{1}$. Quando há necessidade de ressecção de parte considerável da parede torácica, é necessária a reconstrução da parte esquelética para evitar a respiração paradoxal, preservando a complacência pulmonar e também a função de barreira de proteção dos órgãos intratorácicos. Para tanto, em algumas ocasiões, é preciso a ação conjunta do cirurgião plástico e do cirurgião torácico para proceder ao tratamento adequado².

O objetivo dos autores foi fazer uma revisão da literatura sobre a abordagem atual dos defeitos adquiridos mais freqüentes da parede torácica.

Para encontrar artigos que pudessem contribuir para uma revisão e atualização sobre as causas mais freqüentes dos defeitos adquiridos da parede torácica e das técnicas atuais para correção desses defeitos foi acessado o sítio especializado de busca www.pubmed.gov. Os autores utilizaram os unitermos "Thoracic wall; Reconstructive surgical procedures; Surgical flaps" simultaneamente e houve retorno de 1377 citações, sendo consideradas para análise 834 citações porque a busca foi limitada ao período de 1996 a 2008. Cada artigo foi avaliado com base no título e no 'abstract'. Os artigos de apresentação de casos foram excluídos porque o escopo dos autores deste texto foi o de encontrar artigos que relatassem as técnicas de abordagem já consagradas. A grande maioria dessas citações não foi aproveitada porque eram artigos que se referiam a defeitos congênitos da parede torácica, também não pertinentes ao objetivo deste trabalho, que pretendeu enfocar somente os defeitos adquiridos. Alguns artigos, apesar de serem considerados pertinentes, não foram incluídos porque cinco deles foram escritos na língua japonesa, três na língua alemã, um na língua lituana e outro na língua chinesa.

\section{de torácica}

Etiologia dos defeitos adquiridos da pare-

Na tabela 1 estão descritos os fatores que provocam direta ou indiretamente defeitos na parede torácica. Entretanto, não raramente ocorrem combinações dessas afecções no mesmo paciente.

Os defeitos mais freqüentes da parede torácica são aqueles decorrentes de infecção. O trauma pode ocasionar a deformidade torácica diretamente ou por propiciar infecções que afetam o tecido lesionado. As neoplasias podem deformar diretamente a parede torácica ou indiretamente quando é necessário ressecção e/ou radioterapia para tratamento das neoplasias. Adicionalmente, a remoção de tecidos internos à parede torácica, como ressecções pulmonares, podem evoluir com infecção e fístulas broncopleurais, obrigando à ressecções costais e musculares.

As infecções, além de mais freqüentes, são a causa que mais influencia na morbi-mortalidade. Podem ocorrer nos tecidos afetados da parede torácica ou, principalmente, no pulmão e cavidade pleural em decorrência de contusão pulmonar, atelectasias secundárias à restrição ventilatória provocada pela dor e hemotórax entre outros fatores. Estas situações podem evoluir para empiema pleural, cujo tratamento costuma ser difícil. Outras vezes, o empiema pleural é decorrente de ressecções pulmonares para tratamento das neoplasias. Para se conseguir a cura do empiema pleural em alguns casos é necessário fazer uma pleurostomia que consiste na abertura ampla da parte da cavidade pleural preenchida com líquido purulento ao exterior para facilitar a drenagem de pus. Com este procedimento em geral se consegue a cura da infecção, mas pode restar uma deformação na parede torácica para posterior correção.

Trabalho realizado no Departamento de Cirurgia da Faculdade de Medicina de Jundiaí/SP- BR.

1. Professor Adjunto de Cirurgia da Faculdade de Medicina de Jundiaí - Jundiaí-SP-BR. 2. Professor Adjunto Colaborador de Cirurgia Torácica da Faculdade de Medicina de Jundiaí - Jundiaí-SP-BR. 3. Professor Associado de Cirurgia Torácica da Faculdade de Medicina de Jundiaí- Jundiaí-SPBR. 
Tabela 1 - Fatores que promovem defeitos da parede torácica.

Infecção
Trauma
Neoplasias
Afecção da parede por contigüidade

Necrose de parte da parede por radiação

\section{Empiema / Pleurostomia}

Osteomielite de esterno ou de costelas

Desalinhamento e/ou lacerações das estruturas da parede

Primárias da parede torácica

\author{
- Câncer de pulmão \\ - Câncer de mama \\ - Metástases para ossos da parede: \\ $\checkmark$ Mama; Rins \\ $\checkmark$ Cólon; Tireóide \\ $\checkmark$ Sarcomas \\ Radioterapia
}

A lesão por radioterapia produz cicatrizes e disfunção tecidual, que também requerem debridamento e reconstrução. Grandes ressecções de tumores da parede torácica, ressecções pulmonares ou de massas mediastinais, como também os defeitos criados por trauma podem necessitar de reconstrução.

Sendo os defeitos adquiridos da parede torácica na maioria das vezes decorrentes de infecções, a base para correção desses defeitos, além de drenagem do pus, é levar tecidos bem vascularizados para essas regiões infectadas. Esses tecidos bem vascularizados vão preencher o espaço morto e permitir a chegada de leucócitos, anticorpos e antibióticos à região infectada.

\section{Empiema torácico primário}

Na fase crônica do empiema há formação de uma carapaça ao redor do pulmão que fica limitado pela cavidade empiemática. Muitas vezes para se tratar o empiema crônico é necessário fazer uma pleurostomia. A pleurostomia deixa um defeito na parede torácica que precisa ser corrigido, às vezes, com rotação de tecido vascularizado para esta região.

\section{pulmonar}

Empiema torácico secundário a ressecção

O empiema torácico pós-ressecção pulmonar pode ocorrer precoce ou tardiamente. A literatura aponta que em aproximadamente $80 \%$ dos empiemas pósressecção pulmonar a cura pode ser obtida com procedimento de pleurostomia. Assim, quando se obtém a cura do processo de formação purulenta, é o momento para se fazer uma reconstrução da parede torácica com rotação muscular. Outras vezes, em alguns casos de ressecção pulmonar, há persistência de uma fístula bronco-pleural, e nesse caso quando esta fístula não se fecha pode ser necessário o reforço da sutura brônquica com rotação de músculos da parede torácica. Nesse caso, a função do retalho muscular é levar tecido bem vascularizado para a região da fístula e reforçar a sutura.

Para se fechar o orifício da pleurostomia é necessário a rotação de um grande músculo para obliteração da cavidade empiemática. O músculo grande dorsal deve ser inicialmente dividido em duas "tiras", sendo uma delas utilizadas para obliteração desse espaço e a abordagem deve ser realizada por uma toracotomia póstero-lateral. Os músculos peitorais maior e menor podem ser usados no lugar do grande dorsal por já serem naturalmente individualizados ${ }^{3}$.

\section{Esterno infectado}

Infecções do osso esterno se tornaram freqüentes após a introdução da esternotomia mediana para operações cardíacas. A osteomielite do osso esterno tem uma incidência ao redor de 1-2\% após operações cardíacas, e tem processo de tratamento prolongado levando os pacientes a um sofrimento longo. Se o processo de osteomielite é mais localizado, ele se cura com debridamento e antibioticoterapia. Outras vezes o processo infeccioso é de maior magnitude, destruindo quase totalmente o osso esterno e levando a uma prejudicial instabilidade da parede torácica. Nesses casos de maior magnitude o tratamento por debridamento e antibioticoterapia resulta em uma alta incidência de morbi-mortalidade, além de estadia hospitalar prolongada. Nessas circunstâncias o tratamento mais eficaz é a transferência para o mediastino de retalhos musculares ou fazer uma rotação do omento do abdome para a região do osso esterno. Para as feridas esternais o retalho muscular mais comumente usado é o músculo peitoral maior e o suprimento sanguíneo para este retalho vem das artérias perfurantes intercostais, ficando a transposição do omento reservada para tratamento das grandes feridas esternais ${ }^{4}$. Nesse caso o omento é uma excelente alternativa devido ao seu excelente suprimento sanguíneo proveniente dos vasos gastroepiplóicos, levando a ótimos resultados em casos de grandes áreas infectadas 5 .

\section{torácica}

\section{Indicações para reconstrução da parede}

A reconstrução da parede torácica compreende tanto a abordagem da parte esquelética como dos tecidos moles. Para quase todos os defeitos da caixa torácica, o fechamento primário, se possível, é o método mais simples e adequado. A localização e o tamanho do defeito são os fatores da maior importância para decisão da abordagem 
a ser feita. Se a deformidade causada pela ressecção envolve parcialmente a parede torácica, o fechamento primário com ou sem enxerto cutâneo satisfaz tanto do ponto de vista estético como oncológico ${ }^{6}$. Quando surge a necessidade de se estabilizar a parede torácica podem ser usados retalhos musculares ou miocutâneos, ou ainda o uso de material sintético, sendo importante recobrir este material sintético com tecido frouxo vascularizado.

A abordagem da parte esquelética pode ser desnecessária nas seguintes circunstâncias: lesão com menos de $5 \mathrm{~cm}$ de diâmetro, limitada a uma ou duas costelas vizinhas; lesão localizada no ápice da caixa torácica; lesão posicionada na região subescapular de até de $7 \mathrm{~cm}$ de diâmetro (exemplo, após ressecção de tumor de Pancoast) ${ }^{5,7}$. Outros autores consideram que o parâmetro a ser usado para reconstruir a parte esquelética deve ser o número de costelas ressecadas ${ }^{8}$. Nesse caso, quando houver ressecção de menos de quatro costelas pode ser necessário o uso da malha de Marlex, Vicryl ou GoreTex, e quando a ressecção for de quatro ou mais costelas deve-se usar o "sanduíche" com uma das malhas mencionadas entremeada com cimento de metil metacrilato ${ }^{2}$. No caso da existência de neoplasias deve-se avaliar a extensão do comprometimento pulmonar e, se houver envolvimento da parede torácica, ressecar em monobloco. As margens cranial e caudal da ressecção incluem um arco costal acima e outro abaixo, numa extensão de 4 a $5 \mathrm{~cm}$ de tecido macroscopicamente normal, sendo as bordas avaliadas pela congelação.

Os tumores do esterno são avaliados da mesma forma que aqueles das costelas, levando-se em conta o provável envolvimento das estruturas vizinhas e a ressecção deve ser feita deixando margens livres de pelo menos $3 \mathrm{~cm}^{9}$. Na maioria das vezes são doenças malignas e, portanto, a ressecção deve englobar 2 a $4 \mathrm{~cm}$ das costelas, além do esterno comprometido e das estruturas vizinhas, se necessário. Não se deve esquecer a preservação dos vasos epigástricos superiores no sentido de manter a viabilidade dos músculos retos abdominais que poderão ser usados na reconstrução das partes moles.

Grandes defeitos da parede decorrentes de ressecção de várias costelas ou ressecção de grande parte do esterno podem deixar um segmento de tórax vulnerável à movimentação paradoxal durante a respiração. Esses defeitos se beneficiam com a utilização de um método adequado para estabilização da parede torácica, promovendo diminuiçã̃o da incidência de complicações pulmonares $^{10}$.

\section{Planejamento Cirúrgico}

Os seguintes itens devem ser levados em conta no planejamento cirúrgico ${ }^{1,11}$ : 1. localização e extensão do comprometimento da parede torácica; 2.condições locais dos tecidos, como por exemplo, irradiação prévia, infecção localizada, inflamação ou cicatriz prévia; 3.estado clínico do paciente, levando se em conta se ele está em uso de quimioterápicos ou corticosteróide; 4 .finalidade do procedimento, isto é, se a reconstrução da parede tem finalidade curativa ou paliativa;

\section{torácica}

\section{Técnicas de reconstrução da parede}

A reconstrução da parede torácica se baseia em três princípios fundamentais: obliteração de cavidades e espaços residuais com retalhos de tecidos bem vascularizados, estabilização da parte esquelética da parede quando ocorrem defeitos maiores no arcabouço ósseo que podem levar a respiração paradoxal, e cobertura dos defeitos também com tecidos bem vascularizados.

\section{e miocutâneos ${ }^{4,12}$}

a) Reconstrução com retalhos musculares

A localização e o diâmetro do defeito da parede torácica são os fatores que determinam a escolha do retaIho a ser usado ( Tabela 2).

Para uso adequado de retalhos musculares e músculo-cutâneos é necessário por parte do cirurgião o conhecimento da anatomia vascular dos músculos da parede torácica, uma vez que interessa fazer a rotação de retaIhos bem vascularizados. O suprimento sanguíneo da parede anterior do tórax é feito pelas artérias torácicas internas direita e esquerda e pelo sistema epigástrico profundo. Esse sistema conecta os vasos maiores do pescoço com os vasos maiores da região inguinal. $O$ entendimento do suprimento sanguíneo colateral através do eixo acrômio-torácico também é importante porque esse suprimento colateral pode ser necessário na reorganização da circulação após a rotação do retalho muscular. Também é importante na técnica de preparo dos retalhos o conhecimento da inervação dos músculos que serão utilizados. Na maioria dos casos é possível fazer a rotação do retalho com manutenção ou pouca alteração da função do músculo envolvido se durante o

Tabela 2 - Retalhos musculares usados preferencialmente de acordo com a localização do defeito da parede.

\begin{tabular}{|c|c|}
\hline Localização do defeito & Retalho preferencial \\
\hline \multicolumn{2}{|l|}{ Parede anterior } \\
\hline \multirow[t]{3}{*}{ · Linha média } & Peitoral Maior \\
\hline & Reto Abdominal \\
\hline & Omento \\
\hline \multirow[t]{4}{*}{ Parede ântero-lateral } & Grande Dorsal \\
\hline & Reto Abdominal \\
\hline & Transverso \\
\hline & Omento \\
\hline \multirow[t]{3}{*}{ Parede póstero-lateral } & Grande Dorsal \\
\hline & Trapézio \\
\hline & Enxerto Livre \\
\hline \multicolumn{2}{|l|}{ Parede posterior } \\
\hline \multirow[t]{2}{*}{ · Parte superior } & Trapézio \\
\hline & Grande Dorsal \\
\hline \multirow[t]{2}{*}{ - Parte inferior } & Grande Dorsal Reverso \\
\hline & Para-espinhoso \\
\hline \multirow[t]{5}{*}{ Defeitos intra-torácicos } & Serrátil \\
\hline & Omento \\
\hline & Grande dorsal \\
\hline & Peitoral maior \\
\hline & Reto abdominal \\
\hline
\end{tabular}


preparo do retalho houver cuidado para preservação da inervação.

O retalho parcial de pele é mais utilizado na prática, porém o retalho total de pele deve ser o preferido para reconstrução de áreas maiores, entretanto, há limitação para uso desses enxertos devido à vascularização inadequada.

\section{b) Reconstrução com enxertos ósseos ${ }^{5}$}

Os enxertos ósseos são reconhecidos como duráveis e bem sucedidos. As desvantagens no uso de costelas como enxertos ósseos é a dor e instabilidade da parede torácica no local da retirada. Às vezes a retirada de enxertos de costelas implica em criar uma nova área de instabilidade da parede em pacientes com a função pulmonar já comprometida. Outros locais para retirada de enxertos ósseos são crista ilíaca, fíbula e tíbia. As considerações a serem feitas sobre esses enxertos são limitação do tamanho do enxerto a ser retirado e dor no local da retirada. Tem havido uma mudança, com tendência atual em preferir o uso de substâncias sintéticas ou invés de enxertos autógenos pelos motivos relatados acima.

\section{c) Reconstrução com material sintético}

A necessidade para reconstrução esquelética da parede do tórax depende do tamanho e da localização do defeito. A reconstrução é necessária para o esterno, parede anterior e lateral, enquanto que defeitos da parede posterior podem não necessitar de reconstrução se recobertos pela escápula e sem risco de rotação interna da mesma ou se o defeito pode ser recoberto pelos músculos posteriores ${ }^{9}$.

Os defeitos com diâmetro maior que $5 \mathrm{~cm}$ costumam comprometer a dinâmica respiratória, sendo obrigatória uma correção que restabeleça a estabilidade estrutural da parede. Isto é, falhas maiores no arcabouço esquelético podem levar a uma acentuada retração inspiratória no local do defeito da parede (respiração paradoxal) com grande prejuízo à dinâmica respiratória. Assim, às vezes, é necessária a utilização de material sintético que, fixado à parede torácica, permite a estabilização dessa parede obliterando o defeito existente e impedindo assim a ocorrência de respiração paradoxal. Alguns autores concordam com a indicação para estabilização da parede quando a lesão da parede é maior que $5 \mathrm{~cm}$, mas também tomam como indicação defeitos maiores que $10 \mathrm{~cm}$ posteriores à ressecção ${ }^{10}$. Na escolha do material a ser usado os fatores importantes a serem considerados são durabilidade, adaptabilidade, que o material cause nenhuma ou mínima reação inflamatória e seja resistente à infecção e tenha transparência ao raio X (para não impedir a análise radiológica do pulmão subjacente) ${ }^{13}$. Obviamente, qualquer material sintético só pode ser utilizado se não houver infecção local.

Os materiais sintéticos que podem ser usados são nylon, silicone, acrílico, silastic, tela de Prolene ${ }^{\circledR}$, tela de Vicryl®, GoreTex® e tela de Marlex®. A tela de Marlex é fácil de manusear, bem tolerada e não apresenta reações de corpo estranho, sendo incorporada pela parede torácica porque permite o crescimento do tecido do paciente dentro da malha. Quando o defeito a ser reconstruído é maior, pode-se usar uma composição de tela de Marlex ou Gore-
Tex com cimento metilmetacrilato (sanduíche de metil metacrilato) 3,5,14. Nesse caso o procedimento consta da colocação da tela de Marlex dobrada, aplicação de uma camada de cimento coberta por outra lâmina de tela de Marlex. Depois, é feito a fixação das bordas dessa tela às bordas do defeito da parede torácica. Como a tela é maleável, o cimento é aplicado para enrijecer. Quando o cimento endurece, confere a essa combinação de materiais a rigidez suficiente para proporcionar a estabilidade à parede torácica semelhante àquela proporcionada pelo arcabouço ósseo. Um estudo mostra que após reconstrução com sanduíche de metil metacrilato ocorrem movimentos respiratórios concordantes da parede torácica reconstruída comparada com o lado são ${ }^{15}$, Isto é, não ocorrem movimentos paradoxais porque o uso de material sintético endurecido proporciona estabilidade à parte da parede que foi ressecada. Gore-Tex tem sido considerado melhor que a tela de Marlex por alguns autores devido à sua melhor maleabilidade, flexibilidade e impermeabilidade, sendo também usado isoladamente ou em composição com o cimento metilmetacrilato à maneira de aplicação da tela de Marlex ${ }^{14}$. O uso de material protético na reconstrução da parede torácica não significa aumento no risco de infecção, desde que os tecidos adjacentes estejam bem vascularizados $^{12,14}$

As figuras 1-3 detalham casos de tumores de parede torácica ressecados com ou sem necessidade de reconstrução da parede.

\section{CONSIDERAÇÓES FINAIS}

Os defeitos adquiridos da parede torácica são decorrentes de infecção, trauma, ressecção de tumores ou devido à radionecrose.

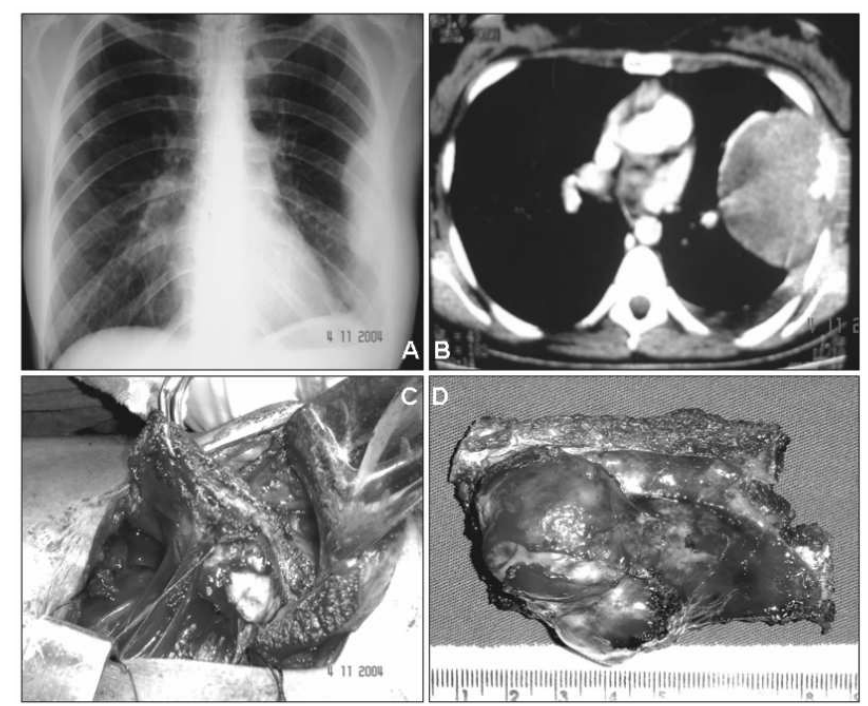

Figura 1 - Radiografia (A) e tomografia (B) do tórax em paciente com tumor de Ewing de parede torácica esquerda. Aspecto do campo operatório (C) e da peça cirúrgica (D). Neste caso, houve necessidade de ressecção dos três arcos costais e reconstrução com tela de Marlex. 

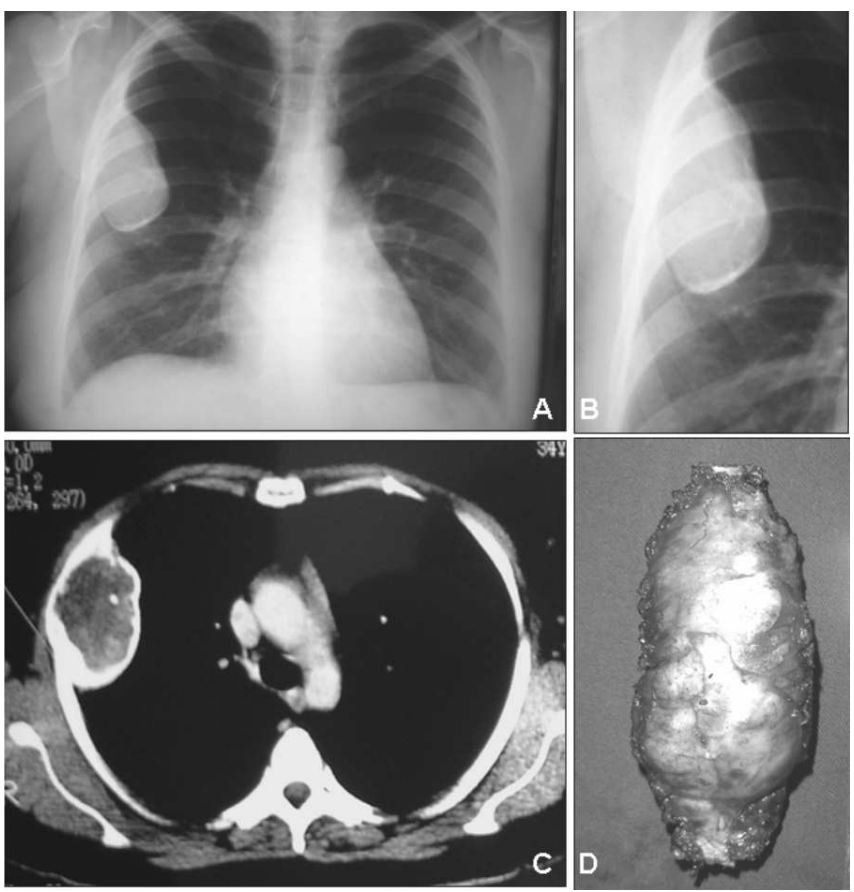

Figura 2 - Radiografia $(A, B)$ e tomografia (C) de tórax de paciente portador de dor torácica crônica em hemitórax direito. Realizada ressecção da lesão (D), que revelou tratar-se de cisto ósseo aneurismático do $4^{\circ}$. arco costal. Neste caso a reconstrução da parede foi feita com os músculos da parede anterolateral.

Baseados nos dados da literatura consultada, concluimos que: 1.A escolha do retalho mais adequado a ser usado para reconstrução da parede é baseada na localização e nas características do defeito a ser tratado; 2.A base para utilização dos retalhos é o conhecimento da vascularização desses retalhos, porque a irrigação vascular precisa ser preservada na ocasião da rotação dos mesmos; 3.Nos defeitos maiores da parte esquelética é necessário a estabilização da parede torácica para evitar a respiração paradoxal; 4.A reconstrução da parte esquelética da parede torácica tem mudado, com ten-
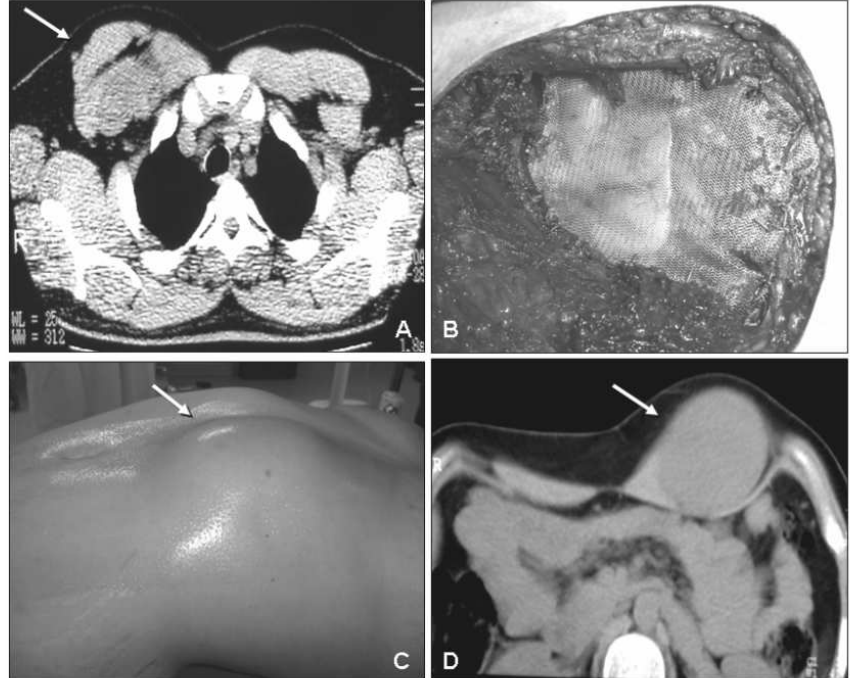

Figura 3 - Tomografia de tórax com tumor fibroso extenso de parede torácica anterior(A, seta). Apesar de benigno, a lesão não tinha planos definidos com os arcos costais. Foto do campo operatório, onde foram ressecados três arcos costais com reconstrução com tela de márlex e rotação muscular (B). Outro caso de lipoma benigno (C) com planos bem definidos na imagem tomográfica (D). Neste caso não houve necessidade de reconstrução da parede.

dência de substituição dos enxertos ósseos por material sintético; 5.0 uso de material protético na reconstrução da parede torácica não tem aumentado a ocorrência de infecção, desde que os tecidos adjacentes estejam bem vascularizados.

\section{Agradecimento}

Os autores agradecem ao Professor Roberto Anania de Paula (Professor Titular de Cirurgia da Faculdade de Medicina de Jundiaí) pelas sugestões e revisão do texto.

\section{A B S T R A C T}

Acquired chest wall defects present a challenging problem for thoracic surgeons. Many of such defects can be repaired with the use of local and regional musculocutaneous flaps, but larger defects compromising skeletal structure require increasingly sophisticated reconstructive techniques. The following discussion will review the options for repair acquired chest wall defects based in literature. The authors searched the Pubmed (www.pubmed.com) and found citations from January 1996 to February 2008. By reading the titles and the abstracts most of the citations were discharged because they focused in congenital chest wall defects or were cases report. However, many papers were found describing the outcome of large series of patients with acquired chest wall deformities. A review of recent literature shows that the repair of chest wall defects with soft tissues, if possible, remains the treatment of choice. Large chest wall defects require skeletal reconstruction to prevent paradoxical respiration. The selection of the most appropriate flap is primary dictated by the location and the size of the defect. It is important to transfer tissue with good vitality, so understanding the vascular supply is imperative. Autogenous grafts have been used in the past for skeletal reconstruction but a combination of synthetic materials with musculocutaneous flaps has been used lately. Based in the literature, the use of prosthetic material in chest wall reconstruction does not significantly increases the risk of wound infection.

Key words: Thoracic wall; Reconstructive surgical procedures; Surgical flaps. 


\section{REFERENCIAS}

1. Ninkovic M, Schoeller T, Schmid T, Salzer GM, Scougall P, Wechselberger $\mathrm{G}$, Ander $\mathrm{H}$. Closure of complex defects in the chest wall with muscle flaps. Scand J Plast Reconstr Hand Surg. 1998;32(3):255-64.

2. Novoa N, Benito P, Jiménez MF, Juan A, Luis Aranda J, Varela G. Reconstruction of chest wall defects after resection of large neoplasm: ten-year experience. Interact Cardiovascular Thorac Surg. 2005;4(3):250-5. Epub 2005 Mar 30.

3. Newsome RE, Jaffer AS, Chiu ES. Chest reconstruction, chest wall reconstruction [Internet]. Emedicine; 2009. Available from: www.emedicine.com/plastic2006/topic458.htm

4. Phillip AG, Pairolero $P$. Chest wall reconstruction: an account of 500 consecutive patients. Plast Reconst Surg. 1996;98(5):804-10.

5. Incarbone M, Pastorino V. Surgical treatment of chest wall tumors. World J Surg. 2001:25(2):218-30.

6. Hultmann CS, Culbertson JH, Jones GE, Losken A, Kumar AV, Carlson GW, Bostwik J, Jurkiewicz MJ. Thoracic reconstruction with the omentum: indications, complications, and results. Ann Plast Surg. 2001;46(3):242-9.

7. Deschamps C, Tirnaksiz BM, Darbandi R, Trastek VF, Allen MD, Miller DL, Arnold PG, Pairolero PC. Early and long-term results of prosthetic chest wall reconstruction. J Thorac Cardiovasc Surg. 1999; 117(3):588-91; discussion 591-2.

8. Chang RR, Mehrara BJ, Hu QY, Disa JJ, Cordeiro PG. Reconstruction of complex oncologic chest wall defects: a 10-year experience. Ann Plast Surg. 2004; 52(4):471-9; discussion 479.

9. Chapelier AR, Missana MC, Couturaud B, Fadel E, Fabre, D, Mussot $S$ et al. Sternal resection and reconstruction for primary malignant tumors. Ann Thorac Surg. 2004;77(3):1001-6; discussion 1006-7.
10. Weyant MJ, Bains MS, Venkatraman E, Downey RJ, Park BJ, Flores RM et al. Results of Chest Wall Resection and Reconstruction with and without Rigid Prosthesis. Ann Thorac Surg. 2006;81(1):27985.

11. Cohen M, Ramasastry SS. Reconstruction of complex chest wall defects. Am J Surg. 1996; 172(1):35-40

12. Skoracki RJ, Chang DW. Reconstruction of the chest wall and thorax. J Surg Oncol. 2006; 94(6):455-65.

13. Mansour KA, Thourani VH, Losken A, Reeves JG, Miller JI, Carlson GW, Jones GE. Chest wall resections and reconstruction: a 25 year experience. Ann Thorac Surg. 2002; 73(6):1720-5; discussion 1725-

14. Sabanathan S, Shah R, Mears AJ, Richardson J. Chest wall resection and reconstruction. Br J Hosp Med. 1997;57(6):255-59.

15. Lardinois D, Müller M, Furrer M, Banic A, Gugger M, Krueger T, Ris $H B$. Functional assessment of chest wall integrity after methylmethacrylate reconstruction. Ann Thorac Surg. 2000; 69(3):919-23.

Recebido em 28/10/2008

Aceito para publicação em 20/12/2008

Conflito de interesse: nenhum

Fonte de financiamento: nenhuma

\section{Como citar esse artigo:}

Carvalho MVH, Rebeis EB, Marchi E. Reconstrução da parede torácica nos defeitos adquiridos. Rev Col Bras Cir. [periódico na Internet] 2010; 37(1). Disponível em URL: http://www.scielo.br/rcbc

\section{Endereço para correspondência:}

Marcus V. H de Carvalho

E-mail: marcus.carvalho@sbccv.org.br 